
\title{
28 Research Square \\ Explaining the Challenges of Coping \\ with Coronavirus Crisis in the Workplaces: A \\ Qualitative Study
}

\section{Abdolhamid Tajvar}

Hormozgan University of Medical Sciences School of Health

\section{Zahra Hosseini}

Hormozgan University of Medical Sciences School of Health

\section{Mohammadreza Farahbakhsh \\ Kerman University of Medical Sciences}

\section{Anahita Fakherpour}

Shiraz University of Medical Sciences

atefeh homayuni ( $\sim$ atefeh_0913@yahoo.com )

Hormozgan University of Medical Sciences School of Nursing and Midwifery https://orcid.org/00000002-4678-7035

\section{Research}

Keywords: Challenge, Coping, Coronavirus, Qualitative study, Workplace

Posted Date: December 28th, 2021

DOI: https://doi.org/10.21203/rs.3.rs-1187068/v1

License: (c) (i) This work is licensed under a Creative Commons Attribution 4.0 International License. Read Full License 


\section{Abstract}

Background: Workplaces play a highly important role in controlling or spreading the prevalence of COVID19. Therefore, the lack of proper implementation of hygienic protocols in theses environments might increase the risk of getting infected with the COVID-19 among the employees, following the increase of the COVID-19 in the family and community levels. This qualitative study was aimed to explain the challenges of coping with coronavirus crisis in the workplaces.

Methods: The present qualitative study was conducted with a conventional content analysis approach. We used the purposeful sampling with maximum diversity in terms of working processes. Data were collected through semi-structured interviews. Interviews were performed to the point of information saturation. MAXQDA software version 10 was used for data management.

Results: Through the content analysis of the interviews with the participants, we identified 2 main classes and 9 sub-classes. The main classes included problems and limitations related to the intra-organizational factors (job nature, budget allocation problems, individual factors, disorganization, manpower and equipment, and Lack of commitment and insufficient support of managers) and extra-organizational factors (lack of accessibility to valid information, black market, and inter-sectorial coordination problems).

Conclusion: The study findings indicated that organizations and industries face numerous internal and external challenges in coping with the COVID-19 pandemic. Paying attention to the mentioned limitations and attempting to eliminate them; especially, by the governmental organizations, employers, and managers could help in effective confronting the COVID-19 pandemic.

\section{Background}

The first cases of respiratory syndrome with an unknown origin were identified in Wuhan city, Hubei province, China in early December 2019. A novel coronavirus was originally abbreviated as 2019-nCoV by the World Health Organization (WHO). This pathogen was later renamed as severe acute respiratory syndrome coronavirus 2 (SARS-CoV-2) by the Coronavirus Study Group (CSG) and the disease was named coronavirus 2019 (COVID-19) by the WHO. Our knowledge about the transmission of the COVID-19 is day by day increasing. The clinical symptoms of this disease ranged from very mild non-respiratory cases to severe acute respiratory ones, sepsis, organ failure, and death. Also, in some cases, subjects have no clinical symptoms. Two main routes of transmission of the COVID-19 are classified into the respiratory droplets and direct contact. The fine droplets thrown out the carrier's mouth or nose while sneezing, coughing, or even talking could spread the coronavirus (COVID-19) directly (infection of other subjects near/close to carrier) or indirectly (contamination of the surrounding surfaces) (1-5). On February 1, 2020, the WHO introduced the outbreak of the COVID-19 as the global emergency and expressed concern about endangering the worldwide public health (6). The COVID-19 pandemic has so far infected $221,558,882$ people and killed $4,581,981$ worldwide (7). 
The occurrence of global pandemics not only led to the human mortalities and adverse physical and psychological consequences but also resulted in a major crisis in the global economy (8-9). The real costs and damages) financial and personal losses) caused by the global spread of the COVID-19 is still unknown precisely because the disease is poorly controlled and rapidly spreading until now. But it is enough to conceive the financial losses caused by the outbreak of the SARS in 2003, which affected only 8000 people and killed 800 people, were reported approximately 4 billion dollars (8).

In the current critical situation, continual activities of the several service and manufacturing companies would be inevitable. It is necessary that some of these companies increase their activities several times more than normal situation. Therefore, the role of workforces in effective managing the present crisis is vital; so, it is very important to maintain their health status.

In accordance with the orders of the Iranian headquarters confronting the COVID-19, the continuous activities of the occupations with low and medium risks became unimpeded with the bet to complying with the hygienic protocols since April 20, 2020 and a large number of employers of companies and production units were required to attend in workplaces and conduct their duties (10). Considerably, the instructions and guidelines regarding the prevention of the COVID-19 in companies and industries which were developed and released by the Environmental and Occupational Health Center (EOHC), Ministry of Health and Medical Education (MOHME) have been generalized. Besides, because of the varieties of situations in different companies and industries (for example, in terms of size, financial status, geographical location, type of work process, etc.), full implementation of hygienic protocols and instructions is not feasible; to do so, this could increase the transmission risk of the COVID-19 virus among the employees; then, in the workplaces, families, and societies $(11,12)$.

According to the high transmission rate of the COVID-19, and considering the possibility that the COVID19 virus to remain with people for a long time due to genetic recombination (13) and since the activity of some service jobs and industries to provide essential services and meet the people's necessities are inevitable, therefore, identifying the existing problems about the implementation of hygienic protocols in the workplaces could be effective in providing practical solutions in order to reduce the barriers to management of the COVID-19 pandemic and increase the government's preparedness to deal with similar crises. According to above mentioned, the aim of the present study was to determine the challenges of coping with the COVID-19 in the workplaces in Iran.

\section{Materials And Methods}

The current study was conducted using the conventional content analysis approach. The content analysis is a qualitative research technique used to interpret the meaning of the content of data text. The classes coding in the conventional content analysis was adapted directly from the data text (14). The study participants were selected among the occupational health and safety managers and officials of the service and manufacturing organizations and companies who were forced to provide services or production despite the outbreak of COVID-19. These organizations included: power generation 
management company, hospitals, steel factory, food industry, port service company, shipbuilding, the gas refinery and so on. The study purpose was firstly explained to the participants via phone call. If they tended to voluntarily take part in the interview and transfer their information and experiences on confronting the COVID-19 pandemic, a consent form would send to them. After signing the consent form, the interview process was carried out by making phone calls to those participants on a specific day and time. Thus, the participants in the study were selected by purposive sampling technique with the maximum diversity in terms of industry and service types and the sampling process continued until data saturation.

The data collection tools were mainly in-depth semi structured interviews. The purpose of the semistructured interviews which conducted with participants was to identify the problems facing organizations in the context of effective management of the COVID-19 crisis.

Due to the current conditions, to protect the interviewees' health, all interviews were conducted using phone call by an appointment for a period of six months, from July, 2020 to December, 2020.

At the beginning of each session, the interviewer explained how to conduct the interview and what the objectives of the study are. After that, the interviews were recorded with the permission of the interviewees. The interviews began with these questions: "How was your company managed the COVID19 crisis?" and "What problems and obstacles has your company been faced in the fight against COVID19 which have been hindered your effective performance?". Furthermore, the "Follow-up questions" such as "Please explain more" and "Please give an example", "If you have a memory, please tell us", and "When you say ... what do you mean?" were also used as needed.

A total of twelve participants consisted of 8 males and 4 females were interviewed. The duration of each interview varied from 30 to 90 minutes. At the end of each interview, the recorded interviews were implemented, and made ready for coding and analyzing. The data and analyses and data collection were performed simultaneously. The texts of the interviews were read several times and analyzed using the MAXQDA version 10 software. The data were converted into semantic units and repeatedly reviewed, and then coded to reveal the hidden thoughts, ideas, and meanings. After extraction of the initial codes, the codes with similar conceptions or relevant meanings were placed in one group with the same class; also, the researchers used the concepts which representative of each class codes with more abstraction in order to name each class. The analysis process continued until the emergence of the minor and major classes. Thus, the analysis process was continued by adding each interview and classes changing.

Considerably, in order to ensure the trustworthiness of our findings, the suggested method by Lincoln and Guba was applied (15). Accordingly, four criteria including acceptance, validity, confirmability and transferability were considered for evaluation. The data validity and acceptability was conducted by reviewing the handwritten notes and extracted codes from the interviews, colleagues' complementary comments, continuous assessment, and sufficient time allocation. Meantime, the study participants took part in the assessment process and one part of the text along with initial codes were read to them in order 
to determine the required points and express their opinions in the case of the contradictions between these perceptions and their conversations and experiences.

\section{Ethical Considerations}

The present study was approved by the ethical committee of the Hormozgan University of Medical Sciences (approval no. IR.HUMS.REC.1399.073). Prior to the interviews, written consent form was obtained from all participants and efforts were made to obtain the subjects' trust to participate in the study. The purpose of the study was explained to the participants and they were assured that their information completely kept confidential from others and they would not be named in the final description and analysis.

\section{Findings}

After data analysis, 2 main classes and 9 sub-classes representing the existing problems and limitations regarding the effective management of the COVID-19 crisis in the workplaces were determined, which were presented in Table 1.

\section{Table 1}

Main classes and sub-classes extracted from the conducted interviews

\begin{tabular}{|ll|}
\hline Class & Sub-Classes \\
\hline $\begin{array}{l}\text { problems and limitations related to the intra- } \\
\text { organizational factors }\end{array}$ & job nature \\
\cline { 2 - 2 } & budget allocation problems \\
\hline individual factors \\
\hline Disorganization \\
\hline $\begin{array}{l}\text { manpower and equipment } \\
\text { Extra-organizational factors }\end{array}$ \\
$\begin{array}{ll}\text { Lack of commitment and insufficient support } \\
\text { of managers }\end{array}$ \\
$\begin{array}{l}\text { Lack of accessibility to valid information } \\
\text { black market }\end{array}$ \\
\hline inter-sectorial coordination problems \\
\hline
\end{tabular}

In the following, we will describe each of the classes.

\section{Class 1: Problems and limitations related to the intra- organizational factors}


The analysis of interview texts showed that intra-organizational problems and limitations play an important role in the effective management of the COVID-19 crisis. This class refers to the problems and obstacles arising from the organization, including management, supervisors, employees, budget, equipment, qualifications, and job nature and included the following 6 sub-classes:

\section{Class 1-1: Job nature}

Project constraints, time constraints, manpower constraints such as the needs for highly qualified specialists with specific skills to perform certain work processes, and the extent and dispersion of work areas including constrains due to work nature and conditions were considered by the most participants as one of the effective barriers to confronting the COVID-19 crisis in the workplaces. Since special expertise were required for some work processes, thus, the organizations have to employ workforces outside the organization or even the forced use of sensitive and vulnerable workforces in these sectors despite the critical situation caused by the COVID-19 pandemic.

"The unique conditions of civil projects are not such that they could observe the hygienic principles. On the other hand, because most of the personnel of the road construction projects are drivers with high age or being retired; in addition, due to time constraints, we could not take any special action to identify the sensitive and vulnerable subjects" (Participant No.6).

"Sometimes there are some special people who have to come and do a special job in the company. For example, one of the machines breaks down, the company's mechanician is unable to solve it; therefore, we have to ask or take out the mechanician, and take out the serviceman, etc. (Participant No.9).

\section{Class 1-2: Budget allocation problems}

Existing economical problems about taking the required measures such as provision of the sanitizers, disinfectants, and personal protective equipment (PPE) were major problems of the most of the studied organizations and industries. Some participants stated that:

"Due to the coordination problems between the BandarAbbas branch and company's headquarter in Tehran regarding the provision of budget, only once 200 masks made from the common fabrics with no standard size were prepared and distributed among all personnel." (Participant No.4)

"We also offered pedal or eye bathroom faucet; however, the company noted that there is no budget now" (Participant No.3)

\section{Class 1-3: Individual factors}


Most of the study participants expressed that dissatisfaction with the lack of employees' cooperation with the organization in the crisis management. Non-observance of quarantine regulations by personnel, unnecessary travels, non-usage of protective tools and equipment, or improper and inappropriate use of those equipment confirmed the lack of cooperation. Some factors such as low perception, restrictive behavioral beliefs, and low perception of the life-threatening COVID-19 were among all factors contributed to the lack of mutual cooperation. Some participants say regarding this issue:

"I do not believe neither masks/respirators nor gloves are useful. Masks are good, but if I didn't have clients, I wouldn't feel the need for a mask. I never believe in donning the gloves. I mean, one subject wears gloves not to touch his/her face by hand; however, I saw that he/she scratched his/her nose with the same gloves" (Participant No.5).

Some participants declared that the opportunistic employees abused the existing conditions by pretending to lie about getting the COVID-19 and wanted to take advantages of a two-week vacation. In contrast, some employees concealed that they had the symptoms of the COVID-19 because of the fear of losing their jobs or job positions.

"Two of the personnel falsely pretended to get infected with the COVID-19 because they had a sport match and the third one, who had decisively pretended that he got sick and had false symptoms such as coughing" (Participant No.1).

"Many people, even if they suspect to the COVID-19 and need to rest, they must either take leave or seek medical treatment. That's why, extensive efforts should be made to determine the subjects who infected with the COVID-19. Because if a person gets infected with the COVID-19 or has symptoms of the COVID19 , he hides it to escape from quarantine or taking leave" (Participant No.12).

Also, some participants stated that the current situations had made some employees obsessed with the disinfectants.

"We sometimes saw that the person disinfected his/her hands from the elbow to fingertips. We gave a$500 \mathrm{cc}$ disinfectant liquid to employees, some of them said that I should use it all in one shift work" (Participant No.2).

\section{Class 1-4: Disorganization}

Some study participants highlighted that their organizations in some dimensions such as recognition of sensitive and vulnerable people, infected, or suspected people, and necessary predictions for employees' returning to workplace after the Nowruz holiday, did not have any special planning and actions.

"We have two employees with diabetes. We didn't forbid them to come to workplace, because they are smart and cheerful." In fact, neither they do not tend nor we do not forced them not to come to the workplace (Participant N0.7). 
Also, some of them didn't have the necessary knowledge about the necessary measures to manage the crisis situations in some cases.

"I don't know that which filters should be used to clean air conditioners. Is it right to use the alcohol? What should we do?" (Participant No.11).

\section{Class 1-5: Manpower and equipment}

Among all, lack of specialized health personnel, lack of welfare and dormitory facilities such as transportation services and lack of facilities in personnel's camps and dormitories in some industries were obstacles which lead to the problems in the effective management of the COVID-19 crisis based on the study participants' beliefs. In this regard, some participants expressed that:

"There were no colleagues who could scientifically be with me (accompanied and cooperated) in the Health, Safety, and Environment (HSE) unit. Those people who work in our unit were transferred from another units to the HSE, and I am the only one who am an expert and proficient and have experiences in my job duties" (Participant No.10).

"There is only one service, everyone rides the same service. If they do not ride, they are left behind; then, they own must return home." (Participant No.2).

"A number of the workforces live in the Conex box (Container), their rooms are small, and they have the least facilities. They do not have specific equipment. They do not have an appropriate locker room" (Participant No.3).

"To carry out the thermometry, I borrowed the thermometer from my friends in other companies. Although thermometry was performed for all personnel, if management had cooperated properly, highly effective actions would have been taken" (Participant No.7).

\section{Class 1-6: Lack of commitment and insufficient support of managers}

Most of the participants in the study pointed out that the insufficient support and inadequate cooperation of management and supervisors of different units were considered as one of the major problems in the management of the COVID-19 crisis. Some factors included not paying attention to the predictions before the arrival of the disease in Iran, not providing sufficient PPE, not requiring the personnel to comply with the health and control principles, not forcing the contractor company by the management to prepare and distribution of the PPE, irresponsibility of the HSE unit, etc.

"We held an emergency committee meeting during the COVID-19 outbreak. After the first session, several sessions held in the HSE office without the presence of top management" (Participant No.3). 
"There was no obligation required by the supervisors of the units to comply with the controlling principles for the personnel" (Participant No.4).

"The only problem was the lack of the management support. Definitely, a formal letter from the manager could be an effective tool for us to be able to implement the company's goals and plans. However, the adequate support unfortunately was not provided regarding this issue or they just asked us to prepare a report. Once, they didn't even check these reports. They did not inspect to ensure whether we carry out the mentioned cases or not" (Participant No.1).

\section{Class 2: Extra-organizational factors}

This class refers to the limitations and problems arising from the factors outside of the organization which includes three sub-classes, namely contradiction and inconsistency in notifying about the information of the COVID-19 outbreak, market-related problems and lack of cooperation and supervision by the deputy of health.

\section{Class 2-1: Lack of accessibility to valid information}

According to some participants' beliefs, other issues that organizations and industries make problems to deal effectively with the COVID-19 virus are the dissemination or distribution of contradictory information, sometimes false information, sporadic training on health tips against the COVID-19 virus, how is the COVID-19 virus spread and how to be safe from the COVID-19 virus in the grouping media, and cyberspace, which confuses many people. In this regard, some participants outlined:

"Many of the staff didn't wear masks at all, despite the given training provided for them. They said the MOHME or the WHO has declared that it was not required to wear a mask in the current situation and donning a mask would be increased the risk of the COVID-19" (Participant No.15).

"Some people believe that the masks equipped with exhalation valves are better than those without exhalation valves because of the inhalation and exhalation. Another people believe that because the masks might be carriers of the COVID-19 virus, prepare those masks without valves. Finally, we didn't know which kinds of masks would be better, because all these information were also stated by experienced professors" (Participant No.12).

\section{Class 2-2: Black market}

According to the most of the study participants, a considerable number of the organizations faced some problems during the outbreak of COVID-19 virus in Iran, including the provision of hygienic items and PPE such as alcohol and masks. Some of the examples of the mentioned problems reported by the participants consisted of the lack of appropriate and standard PPE, low quality of the PPE, high cost of 
the health supplies and PPE at the beginning of the COVID-19 outbreak in Iran and lack of supervision of the Ministry of Industry, Mining, and Trade (MIMT) Institute regarding this issue, existence of the black market and cumbersome rules for provision of the PPE. Some participants claimed that in this regard:

"Our main problem was the provision of the PPE such as masks and disinfectants. Although the management was very persistent in preparation of the health supplies; however, that's why it was a national problem, no company could provide it. We even had communicated and corresponded several times with the health center in order to get access the health supplies to the craftsmen and sold them at the price announced by the health center; however, we could not be successful at providing the health supplies" (Participant No.1).

"Another issue is the quality of masks. Actually, hand-made masks are prepared for us i.e., they don't have good qualities. It should be mention this is not a constant situation, but sometimes the masks are not made of good qualities" (Participant No.10).

"The present cost of the masks is about 35000 Rials, we bought them about 5000 Toman. Whilst, the primary cost of those masks ranged from 2500 to 3000 Rials. The costs of disinfectants have been doubled. The primary cost of thermometer was about 4000000 Rials, its price was approximately 1.5 millions tomans on 10th March, 2021. We didn't buy for those prices; therefore, we have bought it at about 5 millions tomans" (Participant No.8).

"There is a black market in such a way that some people are selling the three-layer surgical masks. We aimed to prevent from the spread of the COVID-19 virus, we have to be content with the some masks that are currently in the markets. Otherwise, these masks are not standard types of the three-layer masks" (Participant No.5).

\section{Class 2-3: Inter-sectorial coordination problems}

Some participants expressed dissatisfaction with, not to address the shortcomings and deficiencies by the deputy of health and their pressures and forces on observing the hygienic principles at the same time. One of the participants remarks regarding this issue:

"We were severely bothered by pressures from the health center. We put under a lot of pressure in the February, during the outbreak of the COVID-19 virus in Iran. We were given a form in excel format, we had to record, document the performed actions, and then, monthly send to the health center. Due to high work load of the HSE personnel, they looked for a series of unexpected actions from the companies. Even though they knew what our shortcomings and needs were; however, the needs were not met and shortcomings were not taken into account" (Participant No.4).

Some participants complained about the lack of proper supervision of the health center on the COVID-19 prevention and control principles. For instance, one of them points out: 
"The district health system (DHS) just asked us to hold a training session and send only some photos to them. They also said that if you want, you could come to get some posters" (Participant No.9).

\section{Discussion}

The study findings were determined in the form of 2 main classes (internal and external organizational problems and limitations) and 9 sub-classes. The analysis of the interview's texts showed that external problems and limitations play an effective role in the management of the COVID-19 crisis. This class refers to the problems and obstacles arising from outside of the organizations or companies. The poor performance of the governments and headquarters confronting the COVID-19 in planning and implementing the emergency response plan, and ultimately the poor management of the COVID-19 crisis was introduced as one of the important sub-classes. Amiri et al. confirmed the poor management of the COVID-19 crisis in Iran and also, the lack of comprehensive and integrated program in the COVID-19 prevention and control considered as one of major shortcomings (16). In another study by Abdollahi et al., the manner and nature of the government decisions on managing the COVID-19 crisis were flawed (17).

Another weakness of the government was attributed to the poor management of the production and supplying of the standard health and safety equipment. This issue led to the employed subjects in the production and service companies had not access to the standard health and safety equipment or had to prepare them by multiple prices higher than the usual prices in which resulted in some problems such as the provision of the budget. Nonetheless, the distribution process of masks and other hygienic products based on the proper and comprehensive planning in some countries such as some East Asian countries (e.g., Taiwan, etc.) has been under full control of the government. This might be one of the reasons for their relative success in fighting against COVID-19 (18). In the research performed by Wong et al. (2020), about one-third of study participants concentrated on the importance of providing the protective equipment including face masks and hand soaps when these protects are not available in the marketplaces (11).

Many occupational health experts participating in the study stressed out the presentation of contradictory information and lack of coordination in notifying the information regarding the COVID-19 by various government agencies and group media such as radio and television, has contributed to the major problems in implementing the hygienic principles in the companies. For example, some of them stated that because it has been announced in the national official media that people in the communities and asymptomatic subjects do not need to use the masks; therefor, many workers refused to wear the masks. While, they were required to wear the masks due to the working conditions. In the study by Abdollahi et al., also criticized the manner of the government in notifying the information about the COVID-19 (17).

Another external organizational problem was the lack of cooperation between health and safety trustees' government agencies with all companies. Many of the study participants stated that they didn't receive any assistance on asking the health deputy experts from the national Universities of Medical Sciences, 
Ministry of Cooperatives, Labour, and Social (MCLS) Welfare, or Ministry of Industry, Mining, and Trade (MIMT) Institute in order to introduce the companies manufacturing the standard equipment. Moreover, another participants claimed that so far the occupational health and safety inspectors or labor inspectors have not monitored the implementation of health protocols in the companies; as a result, the lack of supervision reduces the commitment and support of their companies' managements in the implementing the hygienic protocols and instructions. Even some have been mentioned this issue led to some members of the company's health and safety unit would be reluctant to perform their duties in the fight against COVID-19. Wong et al. concluded that if the government doesn't seriously and precisely monitor the compliance with the hygienic protocols, socially irresponsible behaviors would put all people in the community at risk. Also, the government could play a key role in managing the COVID-19 crisis. On the other aspect, poor performance of the government could cause other problems in the organizations (11).

One of the main classes determined in the present study was the external organizational problems and limitations, which included 6 sub-classes. Restrictions regarding the nature and working conditions of various occupations have resulted in major difficulties to implement the hygienic protocols against the COVID-19 in some workplaces. Some study participants reported that most of the instructions and procedures developed by the MOHME are not appropriate to the type of work processes, job nature, and employees' working conditions in their companies, and many of them are not practically applicable. In the study conducted in the Hong Kong (2020), one-third of the participants suggested that work instructions are required to be revised and updated (11). Therefore, it is recommended that hygienic guidelines and protocols should be developed based on the risk assessment and all job characteristics (12). In the review performed by Webester et al., paying attention to the subjects' jobs to prevent from the spread of the infectious diseases in the community is so crucial (19).

Most of the study participants dissatisfied with the incorporation between the workers and organizations such as not doing self-quarantine at home, unnecessary vacations as much as possible, not usage or irrational and improper usage of the disinfectant products containing alcohol (of around 70 percent) or bleach. According to the study by Wong et al. (2020), only two-third of the study respondents washed their hands with soap before eating foods or after bathroom use. Furthermore, the mentioned study findings showed that the respondents worried about the "leaving without pay" or "legal penalties for absenteeism or compulsory quarantine due to getting infected with the Influenza or COVID-19"(11).

One of the problems disrupting the fighting process against the COVID-19 was the concealment of illness and attendance at workplace by some people who get infected with the COVID-19. The major causes of this issue, they are fear of losing their jobs or due to their employment contracts are on a daily basis; therefore, they are fear if they declared their illnesses openly to the employer, consequently, causing them not to corporate with the organizations. Baker et al. pointed out the accessibility to paid leave varies greatly depending on the occupational position of the worker, type of industry, type of the duties, and financial situation of the company, and some workers due to the type of employment contract are useless from the benefits of leaving with salaries, and this issue leads to hide their illness and attend at the workplace in order to earn a living despite having symptoms. While, attendance of the employees getting 
infected with the COVID-19 is a critical and concerning issue during the COVID-19 epidemic (12). Therefore, employees should be encouraged to stay home when they get infected with the COVID-19 and not to work until improving their health status in order to prevent possibly spreading it to others. The job guarantee and job security and extension of sick leave or paid leave for a longer period are important policies to protect the employee's health during a serious infectious pandemic and considered as a way to motivate the sick employees. In addition, provision of high quality health insurance is especially useful in the current difficult situations.

Some of the study participants also stated that some of the workforce took advantages of the current situation and pretended to be ill in order to take advantages of the two-week quarantine opportunity, and this led to the hard diagnosis of sick employees from non-patient ones; then, both direct and indirect costs are imposed on the company (20).

Another internal organizational problem was the lack of health professionals. Some of the participants highlighted that they are not able to alone implement hygienic protocols. Meanwhile, some participants complained about the authorities who did not expertise in the field of the health in the workplace. Therefore, due to the prolongation of the COVID-19 pandemic. It requires adherence to hygienic principles and protocols of the national headquarters confronting the COVID-19 in all workplaces in order to long term success in confronting the COVID-19. On the other aspect, some suggestions including more seriously investigating and supervising by the inspectors of the Iranian Universities of Medical Sciences. The MCLS should employ occupational health and safety specialists in the companies and request the employers to support them by providing the adequate protective equipment.

Most of the participants pointed out that the insufficient support and inappropriate cooperation of managers, employers, and supervisors are considered as one of the major problems in managing the COVID-19 crisis. One study conducted in Japan (2020), showed that the most important factor influencing the motivation of the health care workers (HCWs) to work in the crisis situations such as epidemics or pandemics, is the ensuring the government support and hospital management, and also trust between the employees and organization. Based on the mentioned study, the best ways to increase the trust between the organization and health service staff during the COVID-19 epidemic are included the following: continuous provision of updated information to staff, continuous communication between the officials and staff and encouragement of the staff, compensation payment on getting infected with the COVID-19 and provision of appropriate protective equipment for the staff (21). Another study concerning this issue which was carried out in Thailand, found that it increases the feelings of confident or trust, social interaction and cooperation between the health professionals, as well as improves the motivation, performance and quality of care services (22). One way to increase the trust between the organization and employees is the provision of frequent updated information to employees. Moreover, frequent communication of government officials and employers with employees and their encouragement creates a sense of security. Paying compensation for getting infected with the COVID-19 could also be an incentive solution to improve the quality of employees' performance. Also, the adequate protective 
equipment and psychological support should also be provided to the staff exposing to the main potential hazards during an epidemic or pandemic.

\section{Conclusion}

Overall, a total of 2 main classes and 10 sub-classes were extracted from the qualitative content analysis. The study findings noted the organizations and industries face several internal organizational challenges consisted of 6 sub-classes (such as job nature, budget allocation, individual factors, disorganization (e.g., lack of proper planning or unplanned), manpower and equipment, and top management support and external organizational challenges consisted of 4 sub-classes (macro policies, accessibility to valid information, black market, and inter-sectorial coordination) in coping with the COVID-19 pandemic.

Paying attention to the mentioned limitations and problems and attempting to rectify them; especially, by the governmental organizations, employers, and managers could help in effective confronting the COVID19 pandemic. One of the best solution is the paying attention to all employees with different positions and their concerns, opinions and experiences; on the other hand, continues communication and cooperation, trust between the employees and top management in order to efficiently and comprehensively solve the progressive problems in the organizations.

\section{Abbreviations}

COVID-19= Coronavirus 2019/ CSG= Coronavirus Study Group/ DHS= District Health System/ EOHC= Environmental and Occupational Health Center/ HCWs= Health Care Workers/ MOHME= Ministry of Health and Medical Education/ SARS-CoV-2= Severe Acute Respiratory Syndrome Coronavirus $2 / \mathrm{WHO}=$ World Health Organization

\section{Declarations}

\section{Ethics approval and consent to participate}

Ethical approval was received for this study from the Ethics Committee of the Hormozgan University of ). Written informed consent was obtained from individualsIR.HUMS.REC.1399.073Medical Sciences ( who participated in this study.

\section{Consent for publication}

Not applicable.

\section{Availability of data and materials}


The datasets used analyzed during the current study are available from the corresponding author on reasonable request.

\section{Competing interests}

All the authors declare that they have no competing interests.

\section{Funding}

This project is funded by a research grant from the Hormozgan University of Medical Sciences. The funding body (HUMS) didn't have any role in the design of the study and collection, analysis, and interpretation of data and in writing the manuscript.

\section{Authors' Contributions}

AT and AH conceptualized and designed the project. AT obtained research funding. AT and AH and led analysis of the transcripts and developed the manuscript. AT, ZH, MF, AF and AH performed the critical review. AT and AH responded to the reviewers' comments. All authors reviewed and approved the final version. Other authors are listed in order of contribution.

\section{Acknowledgements}

The authors would like to acknowledge the financial support of the Hormozgan University of Medical all participants in this research. Sciences. Also, we are grateful to

\section{References}

1. Novel CPERE. The epidemiological characteristics of an outbreak of 2019 novel coronavirus diseases (COVID-19) in China. Zhonghua liu xing bing xue za zhi=. Zhonghua liuxingbingxue zazhi. 2020;41(2):145.

2. Organization WH. Coronavirus disease (COVID-19) advice for the public. 2020.

3. Huang C, Wang Y, Li X, Ren L, Zhao J, Hu Y, et al. Clinical features of patients infected with 2019 novel coronavirus in Wuhan, China. The lancet. 2020;395(10223):497-506.

4. Chan JF-W, Yuan S, Kok K-H, To KK-W, Chu H, Yang J, et al. A familial cluster of pneumonia associated with the 2019 novel coronavirus indicating person-to-person transmission: a study of a family cluster. The Lancet. 2020;395(10223):514-23.

5. Organization WH. Advice on the use of masks in the context of COVID-19: interim guidance, 5 June 2020. World Health Organization; 2020. 
6. Peeri NC, Shrestha N, Rahman MS, Zaki R, Tan Z, Bibi S, et al. The SARS, MERS and novel coronavirus (COVID-19) epidemics, the newest and biggest global health threats: what lessons have we learned? International journal of epidemiology. 2020.

7. WHO. Reported Cases and Deaths by Country, Territory, or Conveyance 2020 [updated September 06. Available from: https://www.worldometers.info/coronavirus/.

8. Jonas OB. Pandemic risk. 2013.

9. Tahir MB, Masood A. The COVID-19 Outbreak: Other Parallel Problems. Available at SSRN 3572258. 2020.

10. updated 20 April 2020. Available from: .

11. Wong EL-Y, Ho K-F, Wong SY-S, Cheung AW-L, Yau PS-Y, Dong D, et al. Views on Workplace Policies and its Impact on Health-Related Quality of Life During Coronavirus Disease (COVID-19) Pandemic: Cross-Sectional Survey of Employees. International journal of health policy and management. 2020.

12. Baker MG, Peckham TK, Seixas NS. Estimating the burden of United States workers exposed to infection or disease: a key factor in containing risk of COVID-19 infection. PLoS One. 2020;15(4):e0232452.

13. Tavakoli A, Vahdat K, Keshavarz M. Novel Coronavirus Disease 2019 (COVID-19): An Emerging Infectious Disease in the 21st Century. ISMJ. 2020;22(6):432-50.

14. Hsieh H-F, Shannon SE. Three approaches to qualitative content analysis. Qual Health Res. 2005;15(9):1277-88.

15. Lincoln YS, Guba EG. Naturalistic inquiry London: Sage Thousand Oaks, CA; 1985.

16. Meskarpour-Amiri M, Shams L, Nasiri T. Identifying and Categorizing the Dimensions of Iran's Health System Response to the Covid-19 Pandemic. Journal Mil Med. 2020;22(2):108-14.

17. Abdollahi A, Rahimi A. Karbarane fazaye majazi: barsakhte ejtemaei korona va siasathaye moqhabele ba an,motaleye moredi. Journal of arzyabi tasirate ejtemayy. 2020;1(2):43-63.

18. Pooladi M, Entezari M, Hashemi M, Bahonar A, Hushmandi K, Raei M. Investigating the Efficient Management of Different Countries in the COVID-19 Pandemic. Journal of Marine Medicine. 2020;2(1):18-25.

19. Webster R, Liu R, Karimullina K, Hall I, Amlôt R, Rubin G. A systematic review of infectious illness Presenteeism: prevalence, reasons and risk factors. BMC Public Health. 2019;19(1):799.

20. Kurgat EK, Sexton JD, Garavito F, Reynolds A, Contreras RD, Gerba CP, et al. Impact of a hygiene intervention on virus spread in an office building. Int J Hyg Environ Health. 2019;222(3):479-85.

21. Imai $\mathrm{H}$. Trust is a key factor in the willingness of health professionals to work during the COVID-19 outbreak: Experience from the H1N1 pandemic in Japan 2009. Psychiatry and Clinical Neurosciences; 2020.

22. Joob B, Wiwanitkit V. COVID-19 in medical personnel: observation from Thailand. J Hosp Infect. 2020;104(4):453. 